\title{
Recent Advances in Computation and Combinatorial Optimization
}

\author{
Xiaodong Hu $\cdot$ Jie Wang
}

Published online: 12 November 2009

(C) Springer Science+Business Media, LLC 2009

This special issue contains six papers selected from 172 submissions to the Fourteenth Annual International Conference on Combinatorics and Computation (COCOON 2008), held in Dalian, China, June 2008. These papers highlight some of the current research interests and achievements in the area of theoretical computer science, ranging from practical exact algorithms for studying complex genetic diseases to improved exact algorithms for classic problems of enumerating cuts, from low-rank matrix factorization and its applications to approximation algorithms for the selected-internal Steiner minimum trees, and from resource bounded frequency computations to quantum separation of local search and fixed point computation.

J. Wang, M. Xie, and J. Chen's paper presents a practical and exact algorithm for studying the individual haplotyping problem on the computational model of Minimum Error Correction with Genotype Information (MEC/GI). Human haplotypes are believed to hold the keys to reveal the mysteries of complex genetic diseases. Computational methods have been explored to tackle challenging issues in the study of human haplotypes. In particular, MEC/GI is a computational model that can be used to infer a pair of haplotypes compatible with the genotype by correcting minimum number of single-nucleotide polymorphisms (SNPs) in the given DNA fragments. The authors develop a dynamic programming algorithm to solve the problem with running time of $O\left(m k 2^{k}+m \log m+m k\right)$, where $m$ is the number of DNA fragments

\footnotetext{
X. Hu (凶)

Institute of Applied Mathematics, Chinese Academy of Sciences, Zhong Guan Cun Dong Lu 55, Beijing 100190, People's Republic of China

e-mail: xdhu@amss.ac.cn

J. Wang

Department of Computer Science, University of Massachusetts, One University Avenue, Lowell, MA 01854, USA

e-mail: wang@cs.uml.edu
} 
and $k$ the number of SNP sites covered by a DNA fragment. While it is NP-hard and hence without efficient algorithms unless $\mathrm{P}=\mathrm{NP}$, the haplotyping problem may still be tractable when $k$ is small. In particular, we note that in real applications with data of low SNP density, we have $k \leq 10$ and so the algorithm presented in this paper can readily be used to obtain exact solutions efficiently.

L.-P. Yeh, B.-F. Wang, and H.-H. Su's paper studies four classic problems of enumerating cuts of graphs by non-decreasing weights, first studied by Vazirani and Yannakakis in 1992, and presents improved algorithms with reduced running time. For the minimum $k$-cut problem with $3 \leq k \leq 6$, for instance, the running time is reduced by a linear factor.

E. Fritzilas, M. Milanič, and S. Rahmann's paper deals with structural identifiability in low-rank matrix factorization. In signal processing and data mining applications, it is often needed to compute, on a given matrix $Y$, two matrices $A$ and $X$ with a low-rank product that provides a good approximation to $Y$; namely, $A \approx A X$. This is a challenging task. The authors provide a solution under the assumption that $A$ is known to have zero values on certain entries. In particular, using the notion of structural rank, the authors present a combinatorial characterization of uniqueness up to diagonal scaling. To demonstrate the usefulness of this result, they study how to use the cheapest subset of sensors to monitor the signal sources while maintaining structural identifiability, where $Y, X$, and $A$ contain, respectively, the sensor measurements over several time samples, the source signals over time samples, and the source-sensor mixing coefficients. The authors show that this is an NP-hard problem. They then present a mixed integer linear programming model to compute an exact solution. They also present incremental algorithms and a greedy approximation algorithm.

X. Li, F. Zou, Y. Huang, D. Kim, and W. Wu's paper studies an interesting variant of the classic Steiner minimum tree problem (SMT) called the selected-internal Steiner minimum tree problem. This variant comes from applications in different fields. It is to find, on a given weighted complete graph $G=(V, E)$ with a weight metric $c$ and two subsets $R^{\prime}$ and $R$ with $R^{\prime} \subseteq R \subseteq V$ and $\left|R-R^{\prime}\right|>1$, a minimum subtree $T$ of $G$ interconnecting $R$ such that $T$ contains no leaves in $R^{\prime}$. The authors present a $(1+\rho)$-approximation algorithm for the problem, where $\rho$ is the best-known approximation ratio for SMT.

U. Hertrampf and C. Minnameier's paper deals with resource bounded frequency computations. Hinrichs and Wechsung conjectured that as soon as the number of inputs to be queried reaches $2^{d}+d$, obtaining an answer with at most $d$ errors does not become harder with more inputs. This conjecture is trivial for $d<3$ and is nontrivial for $d \geq 3$. The major contribution of this paper is to show that the conjecture is indeed true for $d=3$.

X. Chen, X. Sun, and S.-H. Teng's paper studies the quantum query complexity for finding a fixed point of a discrete Brouwer function over grid $[n]^{d}$ and presents a lower bound of $\Omega\left(\sqrt{n^{d-1}}\right)$. This lower bound is nearly tight, as the Grover Search technique can be used to find a fixed point with $O\left(\sqrt{n^{d}}\right)$ quantum queries. This result provides a quantum separation of local search and fixed point computation over $[n]^{d}$ for $d>4$. 